\title{
Advancing regenerative medicine
}

\section{Regenerative medicine may enable replacement of damaged or diseased tissues. But its clinical success will require deeper understanding of the basic biology of the stem cell niche and coordination between stem cell biologists and those in other fields.}

t's been more than 15 years since the term 'regenerative medicine' entered our scientific lexicon. Yet at the recent International Society for Stem Cell Research meeting in Vancouver in June, it became evident that we still do not fully understand the barriers to translation of regenerative medicine-which may differ according to the type of stem cell and therapeutic application-let alone how to overcome them. The dichotomy between the promise of this field and the hindrances it faces is apparent in a series of Reviews and Perspectives that we are proud to publish in Nature Medicine in a joint focus with Nature Biotechnology.

Although some of the Nature Medicine articles review our current knowledge of the development and function of stem cells in different tissues and organs, most reach beyond the stem cells themselves to reflect on the influence of the niche during aging, injury and regeneration. In addition, the role of the host immune response in regeneration is discussed. The Nature Biotechnology pieces cover key considerations for translation such as the biomaterials used for scaffolding or encapsulation, the ability to image and track the localization and persistence of infused stem cells, and the potential contributions of engineering advances such as three-dimensional printing.

Basic stem cell biology may seem removed from clinical considerations, but even incremental steps in our understanding of regenerative biology cannot be ignored. The Review by Edward Morrisey and Darrell Kotton (page 822) on lung regeneration demonstrates that we still have yet to fully understand the developmental biology of the lung or unambiguously identify its potential progenitor or stem cell populations. We face the same challenges in other tissues, as well. The identification of factors responsible for normal differentiation of tissue stem cells could be key in enabling efforts to therapeutically induce the differentiation and mobilization of endogenous stem cells in vivo or to generate and grow stem cells in vitro prior to injection back into a patient. In the lung and other tissues, although we have some knowledge of which molecules and signaling pathways are responsible for stem cell maintenance and differentiation, it is difficult to assign more weight to one factor over another owing to our still-nascent understanding of the nuances of their roles and importance relative to each other. The identification of potentially potent 'master regulators' is paramount to efforts to design small molecules able to mimic their function.

The stem cell niche also needs to be better understood so that we can identify the best strategies for stem cell and organ transplantation. Elaine Fuchs and her colleagues (page 847) discuss the role of the niche in the maintenance and differentiation of skin stem cell populations, and Paul Frenette and Avital Mendelson (page 833) consider the components of the hematopoietic stem cell niche. The niche may also come into play during stem cell aging and pool depletion, as emphasized by Amy Wagers and her colleagues (page 870).

A Perspective highlights various roadblocks facing the safe and effective translation of our current knowledge about the basic biology of the niche and the factors that influence stem cells into the clinic (page 814). In a Review, Stuart Forbes and Nadia Rosenthal (page 857) focus specifically on the ways in which the local microenvironment or niche-be it cellular components or secreted factors-and the systemic environment may dictate the ability of a stem cell to engraft successfully to repair tissue. Clinical trials have shown, for example in the brain (Regen. Med. 8, 145-155, 2013), that, often, few stem cells engraft, and those that do engraft may persist for a limited period of time. Furthermore, within a diseased tissue, the niche may be highly inflamed or aged, but inflammation of the niche may also be necessary to repair damaged tissue and promote tissue regeneration.

In line with the above observations regarding stem cell engraftment, there is an emerging consensus that the limited therapeutic benefit of stem cell-based therapies currently in clinical trials, such as infusions of exogenous mesenchymal stromal cells or epithelial progenitor cells in the lung, cannot necessarily be attributed to tissue regeneration (Mol. Ther. 20, 1116-1130, 2012). Paracrine effects may also be at play. Distinguishing and harnessing the two will require deeper understanding of the cellular and molecular mechanisms and processes underlying the physiological effects of stem cell infusion.

Finally, our understanding of the safety of stem cell-based therapies is also underdeveloped. Many of the factors identified that regulate stem cell maintenance or differentiation, for example in hematopoiesis, are also involved in tumor progression, so it is possible that modulating these for regeneration purposes could inadvertently result in tumorigenesis. For this reason and others, skipping straight to the clinic may not be the right way forward (page 796).

Application of new technologies may be key to revealing the basic insights of and to developing the standards (page 797) needed for successful and safe regenerative medicine. It is partly for this reason that we produced this focus jointly with Nature Biotechnology. We hope our readers find these articles informative and thought provoking, and we welcome your feedback. 